\section{A E E T}

ASOCIACIÓN ESPAÑOLA DE ECOLOGÍA TERRESTRE

\section{ecosistemas}

REVISTA CIENTÍFICA DE ECOLOGÍAY MEDIO AMBIENTE

MONOGRÁFICO: $\quad$ ISSN 1697-2473 / Open access

Vulnerabilidad de las especies al cambio climático disponible en www.revistaecosistemas.net

\title{
Cambio climático y plagas: algo más que el clima
}

\author{
J.A. Hódar ${ }^{1, *}$, R. Zamora ${ }^{1}$, L. Cayuela ${ }^{2}$ \\ (1) Departamento de Ecología, Facultad de Ciencias, Universidad de Granada, E-18071 Granada, España. \\ (2) Área de Biodiversidad y Conservación, ESCET, Universidad Rey Juan Carlos, c/ Tulipán s/n. E-28933 Móstoles, Madrid, España. \\ * Autor de correspondencia: J.A. Hodar [jhodar@ugr.es]
}

> Recibido el 10 de octubre de 2012, aceptado el 28 de noviembre de 2012.

Hódar, J.A., Zamora, R., Cayuela, L. (2012). Cambio climático y plagas: algo más que el clima. Ecosistemas 21(3):73-78. Doi.: 10.7818/ECOS.2012.21-3.09 Hay un acuerdo general en la comunidad científica respecto al efecto que el cambio climático va a significar para las especies plaga: la mayor parte de ellas se van a ver favorecidas, lo que augura un panorama difícil para la sostenibilidad y gestión de muchos de nuestros bosques. Usando como hilo conductor a la procesionaria del pino (Thaumetopoea pityocampa), una plaga circunmediterránea que afecta severamente a los pinares y cuya respuesta al incremento de temperaturas es bien conocida en Europa, señalamos la necesidad de un abordaje más integral para la futura gestión de estas plagas. Frente a los procedimientos de control basados en el ataque directo a la especie plaga, mostramos las posibilidades de una estrategia de prevención basada en el manejo del hábitat, que consiga bosques más diversos en estructura y composición específica, más resistentes y resilientes no sólo frente a las plagas, sino a los diversos tipos de perturbación que se adivinan para los años venideros.

Palabras clave: diversidad y estructura del bosque, manejo del hábitat, plagas, procesionaria del pino, pinar, repoblaciones forestales.

Hódar, J.A., Zamora, R., Cayuela, L. (2012). Climate change and pests: more than climate Ecosistemas 21(3):73-78. Doi.: 10.7818/ECOS.2012.21-3.09 The general consensus in the scientific community about the effects of climate change on pest species is that pests will be mostly favored by the increase in temperatures. This will create a difficult scenario for the maintenance and management of woodlands. In this work we use as an example of this situation the pine processionary moth (Thaumetopea pityocampa), a Mediterranean pest causing severe damages to pine woodlands. This moth already shows a well-known expansion in Europe as a consequence of temperature increase. We propose an integral strategy to face the future management of this pest. Alternatively to control methods based on direct chemical or biological attacks to the insect, we evidence the possibilities of creating prevention strategies based on habitat management. We suggest to increase ecological heterogeneity of pine plantations, which usually have low structural and species diversity. This would result in ecosystems with higher resistance and resilience against not only pests, but also diverse disturbances that would be menacing woodlands in future years.

Keywords: habitat management, pests, pine processionary moth, pine woodlands, woodland diversity and structure, wood plantations.

\section{Introducción}

La información científica de la que disponemos sobre los efectos que el cambio climático tendrá en los sistemas biológicos acumula ya bastantes certezas pero sigue arrastrando algunas incertidumbres. Entre las primeras, una de las que antes fue establecida fue el efecto que el cambio climático tendría en lo que habitualmente conocemos como especies plaga. En una fecha tan temprana como 1993, Matthiew P. Ayres dedicaba un capítulo entero del libro Biotic interactions and global change a las previsibles alteraciones que el cambio climático ocasionaría en las interacciones planta-herbívoro, y analizaba los efectos que dichas alteraciones tendrían en las plagas agrícolas y forestales. El incremento en las temperaturas afecta tanto a los insectos defoliadores como a las plantas, en ambos casos adelantando la fenología y acelerando el metabolismo, pero si la respuesta a ese cambio es más rápida en uno de los interactores, el otro tendrá problemas. Si la planta crece más rápido, los defoliadores encontrarán tejidos más duros para comer en sus fases iniciales de desarrollo, y habitualmente perecerán; si, por el contrario, el insecto responde más rápidamente, las fases iniciales de su desarrollo encontrarán tejidos vegetales poco endurecidos y desarrollados, sobrevivirán bien e infligirán graves daños a las plantas.
Lo que ya se sabía en 1993 era que en general los insectos responden más rápido que las plantas al incremento de temperatura, de modo que lo previsible era un incremento en la frecuencia y la virulencia de las defoliaciones. Desde aquel pionero trabajo de hace casi 20 años, se han escrito varias revisiones sobre el tema (p. ej. Williams y Liebhold 1995, Ayres y Lombardero 2000, Fuhrer 2003, Logan et al. 2003, Kocmankova et al. 2010, Ladanyi y Horvath 2010), llegando en la mayoría de los casos a conclusiones similares a las expuestas por Ayres (1993). El número de trabajos de investigación también ha aumentado de forma notable en los últimos años. Una búsqueda en ISI Web of Knowledge con los términos insect pest climate change temperature ofrece 137 resultados, de los cuales la mitad (67) se han publicado en los últimos tres años (Fig. 1).

La solidez de las conclusiones ofrecidas en los trabajos citados es, a estas alturas, difícilmente rebatible, pero creemos que centrar tanto la atención en componentes específicos del cambio global como el calentamiento global o la fertilización por $\mathrm{CO}_{2}$ ofrece una visión incompleta del problema. Además, esta visión hace olvidar otros posibles métodos de actuación contra las plagas. En este artículo nos basaremos en nuestras investigaciones más recientes sobre una plaga forestal bien conocida en España, la procesionaria del pino (Thaumetopoea pityocampa), que encaja perfectamente 


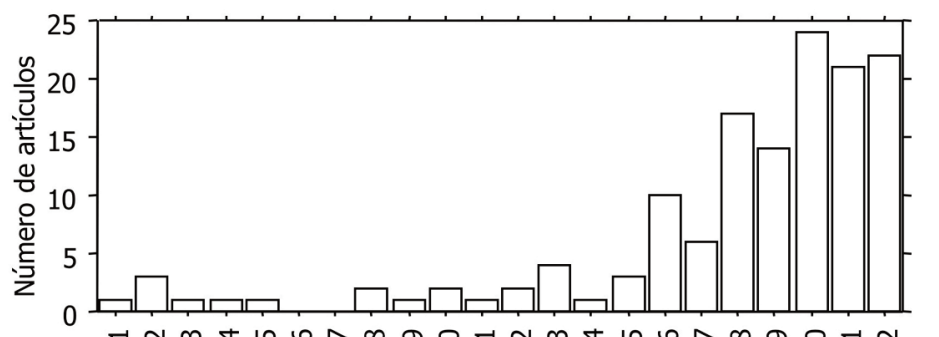

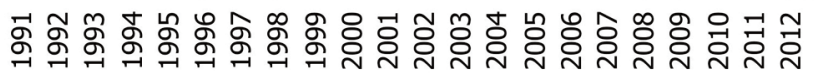
Año

Figura 1. Número de artículos publicados cada año en revistas listadas en ISI Web of Knowledge con los términos insect pest climate change temperature (Octubre de 2012).

en el patrón descrito de respuesta de una plaga al calentamiento global. Describiremos cómo la procesionaria del pino muestra en efecto una notoria respuesta al calentamiento global, nos detendremos en las medidas de control que habitualmente se han usado, evaluando su posible efectividad en un contexto de cambio climático, y finalmente explicaremos las posibilidades de un enfoque alternativo, basado en las características comunes a las especies plaga, para ofrecer una solución diferente a los problemas causados por esta y otras plagas forestales.

\section{Una plaga en expansión}

La procesionaria del pino se ha convertido en un caso paradigmático a la hora de analizar los efectos del cambio climático en las plagas forestales (Battisti 2008, Netherer y Schopf 2010). La larva de la procesionaria se desarrolla durante el invierno, y por tanto, cabe suponer que el incremento de temperaturas tendría en ella un efecto positivo. Pero además, no se trata sólo una cuestión de sanidad forestal, sino también de salud pública, dado que las larvas de procesionaria son urticantes y causan serios problemas sanitarios a la población en riesgo (p. ej. Vega et al. 2011). Por lo tanto, muy desde el principio ha estado en el punto de mira ante el paulatino incremento de las temperaturas.

La relación entre las defoliaciones por procesionaria y la temperatura, en particular los inviernos suaves, ya había sido puesta de manifiesto hace años (ver, por ejemplo, los trabajos clásicos de Démolin 1969a, b), aunque no ha sido hasta recientemente que hemos entendido la forma en la que las bajas temperaturas invernales limitan el desarrollo de las larvas, así como los mecanismos de tolerancia de las larvas frente al frío (Buffo et al. 2007, Robinet et al. 2007, Hoch et al. 2009). En un contexto de calentamiento global era esperable que la procesionaria colonizara zonas que, en razón de su latitud o altitud, hasta ahora le estaban vedadas, y en efecto, en los últimos años varios estudios han confirmado dichos movimientos altitudinales (Hódar y Zamora 2004, Battisti et at. 2005, 2006, Petrucco-Toffolo et al. 2006) y latitudinales (Battisti et al. 2005). Todo indica que la procesionaria es una especie netamente favorecida por la tendencia al incremento de temperaturas observado en los últimos tiempos. En paralelo, se ha encontrado una buena conexión entre las superpoblaciones de procesionaria y variables climáticas a escala local (Hódar y Zamora 2004) y regional (Hódar y Zamora 2009, Hódar et al. 2012a), lo que refuerza esta visión.

En resumen, la procesionaria depende de la temperatura para su desarrollo, hay un ajuste entre clima y defoliaciones, y sus poblaciones se están expandiendo en altitud y latitud.

\section{El diablo está en los detalles: no es solo el clima}

Si realmente aquí está el fin de la historia, poco podemos hacer: el cambio en el clima supondrá un escenario favorable para que la plaga progrese, y salvo que alteremos de nuevo el clima, esta vez a nuestro favor, la plaga no tendrá obstáculos para su avance.
Pero, desde luego, con ser el clima importante, no es lo único que determina la dinámica poblacional de la procesionaria. Así, una de las estrategias más conocidas para combatir a la procesionaria es la de favorecer a sus depredadores (p. ej. aves, parasitoides), en el entendimiento de que son capaces de ofrecer algún tipo de regulación sobre la población (Pimentel y Nilsson 2007, 2009, Barbaro y Battisti 2010). Además, en varias zonas europeas se han descrito ciclos más o menos periódicos de aproximadamente seis años (Robinet 2006, Hódar y Zamora 2009), un ciclo cuya longitud y periodicidad sugieren una regulación por factores bióticos (Berryman 2002). No está, pues, tan claro, que sea el clima el único factor capaz de explicar la dinámica poblacional de esta plaga. ¿Nos ofrece esto una vía alternativa para entender y, en su caso, controlar su dinámica?

Hagamos un inciso y acudamos a la literatura especializada para revisitar la definición de "plaga". Peter Jarvis (2000), un clásico de la ecología aplicada, define las plagas, junto a las enfermedades infecciosas, de la siguiente forma:

"Las enfermedades (refiriéndose también a las plagas) que llegan a ser agudas y estar ampliamente repartidas, epidémicas, lo son cuando: (1) la población del hospedador es susceptible, tanto más cuanto más uniforme (esto es, que los hospedadores son susceptibles por igual y no hay, o hay pocos, individuos resistentes a la enfermedad o plaga), (2) los hospedadores están agrupados o apiñados muy juntos, (3) el patógeno presente incrementa su abundancia con gran rapidez (capacidad reproductiva), (4) el clima y otros factores son favorables a su diseminación y desarrollo, y (5) el periodo de condiciones favorables debe ser suficiente para sostener la epidemia."

$Y$ es que, en efecto, las características de una plaga encajan en general bastante bien con las de una enfermedad infecciosa. Ciñéndonos al caso de la procesionaria, ya hemos visto su dependencia de las condiciones climáticas (puntos 4 y 5). Tiene una alta capacidad reproductiva (punto 3); la puesta promedio es de unos 200 huevos, lo que le permite incrementar su población en dos órdenes de magnitud de un año para otro. También el punto 1 es bastante claro: aunque casi todos los pinos son en cierto grado susceptibles a la procesionaria, las grandes defoliaciones se dan en hospedadores susceptibles, como el pino albar o el salgareño cuando las condiciones son adecuadas (Fig. 2), o de forma crónica en pinos exóticos muy palatables como el canario (Pinus canariensis) o el pino de Monterrey ( $P$. radiata, Cobos-Suárez y Ruiz-Urrestarazu 1990). Esto también puede explicar por qué extensas zonas de pinares en tierras bajas y cálidas, como Doñana (con $P$. pinea) y la costa mediterránea (con $P$. halepensis) no son sistemáticamente defoliadas todos los años, pese a su bonanza térmica invernal para la procesionaria (Hódar et al. 2012a). Por otra

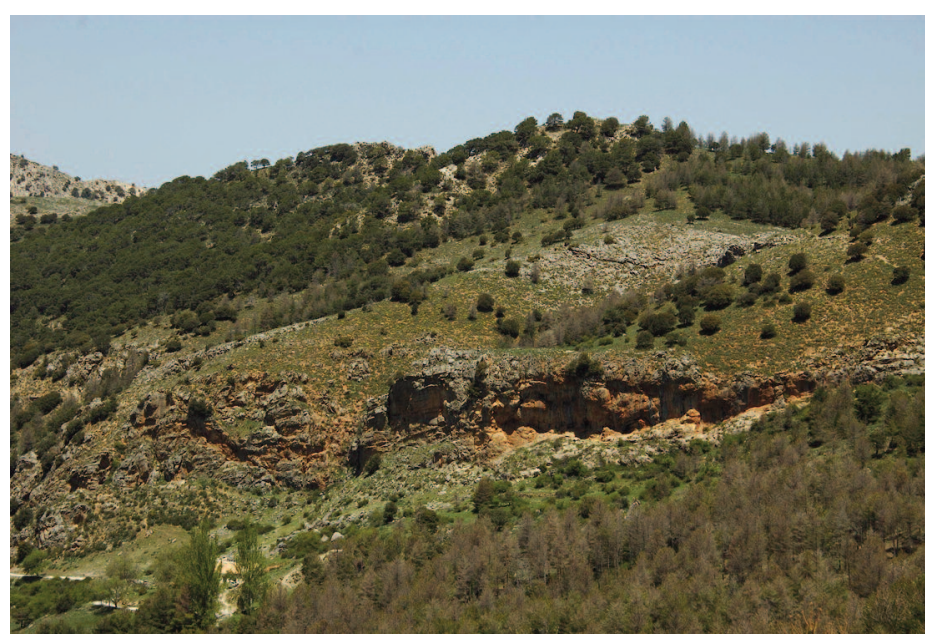

Figura 2. A pesar de las condiciones homogéneas en toda la loma, sólo los rodales de Pinus sylvestris y P. nigra (abajo, derecha) están defoliados, mientras que los de Pinus pinaster (arriba, izquierda) están intactos. Pinar de la Cortijuela del Trevenque, Sierra Nevada, invierno de 2010. 
parte, el piñonero y en menor medida el carrasco son pinos poco susceptibles a la procesionaria, y además las altas temperaturas durante el desarrollo de la larva favorecen la aparición de parásitos y depredadores.

Menos claro podría parecer el punto 2, ya que la procesionaria necesita temperaturas adecuadas para su desarrollo en invierno y normalmente evita formaciones muy densas, situándose preferentemente en los bordes de las masas forestales bien soleadas (Roques et al. 2011). Pero si entendemos la agrupación de los hospedadores a una escala espacial algo más amplia, una cierta continuidad en los pinares favorece la infección de los parches locales cercanos, ya que aunque la mariposa puede eventualmente hacer vuelos de larga distancia, la mayor parte de sus movimientos son de corta o muy corta distancia (Robinet et al. 2012).

Lo más notorio de las características citadas por Jarvis (2000) es que dos de ellas ( 1 y 2 ) son características del medio en el que la plaga se desarrolla, otras dos (4 y 5 ) son factores climáticos, y sólo una de ellas (3) es realmente una característica de la especie. Dicho de otro modo, más que especies plaga, tenemos especies capaces de aprovechar muy a su favor (y en detrimento de otras especies) unas condiciones ambientales propicias para su crecimiento exponencial.

\section{Los métodos tradicionales del control de plagas}

El interés de estos hechos radica en que el control de la procesionaria en particular, y de las plagas en general, se ha basado en gran medida en una ofensiva directa sobre la especie plaga (Schowalter 1986, Kogan 1998), y no tanto sobre el hábitat en el que vive. Esta visión fue especialmente prominente entre 1940 y 1960, un período que ha dado en llamarse "la edad oscura del control de plagas" (Newsom 1980). La aparición de insecticidas organoclorados como el DDT hizo pensar que las plagas quedarían definitivamente controladas y que se podrían exterminar, lo que condujo a un creciente uso de los insecticidas en detrimento de otros métodos de control biológico (Pimentel y Perkins 1980). Esta visión duró apenas hasta mediados de los 60 , cuando la aparición de resistencia a los insecticidas, el resurgir de antiguas plagas y la emergencia de otras nuevas, y los efectos colaterales en otros organismos incluido el hombre, evidenciaron el error de este planteamiento basado exclusivamente en el uso de productos químicos.

Se fue imponiendo entonces la necesidad del control integrado de plagas, que puede definirse como "un sistema de apoyo a la decisión para la selección y uso de tácticas de control de plagas, individuales o coordinadas en una estrategia de manejo, basado en un análisis de costo/beneficio que tiene en cuenta los intereses y el impacto en los productores, la sociedad, y el medio ambiente" (Kogan 1998). Nótese que, definido así, no hay una referencia clara a qué tipo de estrategia es preferible: no hay una preferencia manifiesta de los métodos de control biológico frente a los químicos, y no se descarta en absoluto el uso de insecticidas. De hecho, es notorio que, incluso tras la renuncia al "organoclorado para todo" y el nuevo impulso del control integrado como estrategia de control de plagas, se le sigue percibiendo como "intensivamente agroquímico", a pesar de las voces que reclaman un cambio hacia un control integrado "intensivamente biológico o biointensivo" (Frisbie y Smith 1991). La propia hipertrofia y compartimentalización que han sufrido las diferentes subdisciplinas del control integrado, que ha dado lugar a una escasa comunicación entre los especialistas en distintas estrategias de control integrado, ha podido ser la causante de estos efectos indeseados (Gurr et al. 2004). Incluso en un control integrado biointensivo, se suele pensar más en promocionar al depredador, parásito o enfermedad específicos de la especie plaga como remedios biológicos contra ella que en otras soluciones realmente integrales.

\section{Controlar el hábitat para controlar a la plaga}

Frente a esta tendencia a solucionar los problemas de las plagas atacando sólo a la especie plaga, planteamos una aproxima- ción basada en el manejo del hábitat, aunque somos conscientes de que una solución realmente integral sería aquella que promociona un abordaje ecosistémico al manejo de la plaga, considerando toda la variedad posible de estrategias ecológicas, desde el comportamiento de las plagas hasta los manejos silvícolas (Schowalter 1986). La prioridad debe ser el modelado de un bosque capaz de disminuir la probabilidad de aparición de las plagas, minimizando la intervención humana.

De hecho, ya en el ámbito forestal, trabajos recientes plantean la necesidad de considerar la estructura del bosque (densidad, clases de tamaño, patrón espacial) como un atributo capaz de modular la respuesta de los ecosistemas forestales ante eventos de cambio climático (Kulakowski et al. 2006, Keeton 2006). Se trata de definir, a partir de este conocimiento, los tipos de manejo que minimicen los efectos del cambio climático y favorezcan la productividad del ecosistema en términos de producción, ciclos biogeoquímicos, biodiversidad, valores paisajísticos, etc., frente a perturbaciones a escala local, regional o global. Nuestra propuesta es hacer explícito este planteamiento a otros factores de riesgo, como las plagas forestales. En un escenario de rápido cambio global es prioritario establecer planes de actuación proactivos, que se anticipen a los problemas a los que se enfrentarán las formaciones vegetales, con el objetivo de preservar su diversidad natural y los servicios ecosistémicos que aportan.

Planteémoslo con un ejemplo. Imaginemos un pinar de repoblación que periódicamente sufre plagas de procesionaria. Para nuestro ejemplo es irrelevante el uso de dicho pinar (maderero, recreativo, prevención de erosión, etc.) Sabemos que el pinar sufre defoliaciones importantes cada vez que hay un invierno suave; con una tendencia al incremento de temperaturas, cabe pensar que los manejos para combatir la plaga van a ser cada vez más reiterados, sean soluciones de tipo fumigación, liberación de parasitoides, promoción de depredadores (cajas nido para aves insectívoras), o todas a la vez. El coste va a ser cada vez mayor. En un medio-largo plazo, mantener el pinar tal cual supondrá una cantidad importante de dinero, pero es entendible que el beneficio reportado por el pinar puede, en efecto, compensar tal inversión y seguir ofreciendo un balance positivo. Pero puede que no; un incremento en los costes de las labores de control, una reevaluación de los beneficios del pinar, o una situación de crisis como la actual que obliga a reconsiderar en qué nos gastamos el dinero podría interrumpir la aplicación de tales medidas, dejando sin control a la procesionaria. La solución ideal sería reducir la susceptibilidad del pinar a la plaga, esto es, que nuestra actuación sobre el pinar permitiera que éste fuera, si no inmune a la procesionaria, sí al menos lo suficientemente resistente como para impedir que la plaga supere un estadío de latencia poblacional. No se trata de eliminar a la procesionaria, se trata simplemente de mantenerla por debajo de un límite poblacional que no cause daños que requieran actuaciones adicionales. La intervención sería de mayor impacto (y más cara) en un principio, pero en lo sucesivo no requeriría de nuevas actuaciones o éstas serían mínimas.

Retomemos ahora las características de las especies plaga. La respuesta a los factores climáticos de la procesionaria (4 y 5) ya ha quedado clara, y es evidente que sólo reduciendo la concentración de gases con efecto invernadero en todo el planeta podríamos a largo plazo cambiar la actual tendencia. La capacidad para incrementar sus poblaciones en poco tiempo (3) es inherente a las características reproductivas de la procesionaria, y poco podemos hacer contra ella. La procesionaria seguirá poniendo 200 huevos por puesta de promedio, y podremos reducir su éxito reproductivo liberando parasitoides, poniendo trampas de feromona, cajas-nido o fumigando, pero esto son soluciones caras (que además tendrán que ser cada vez más reiteradas) y cuya eficacia es, como poco, discutible (Cayuela et al. 2011).

Nos quedan las características 1 y 2: la plaga puede desarrollarse cuando la población del hospedador es susceptible, densa y continua en el espacio. Un retrato fidedigno de la mayor parte de nuestros pinares naturalizados o de repoblación, que representan 
un magnífico caldo de cultivo para una plaga. De hecho, sabemos que los bosques más diversos son en general menos susceptibles a las plagas. Aunque sigue siendo un tema de discusión, el consenso en este sentido es amplio y antiguo (ver p. ej. Jactel y Brockerhoff 2007 y referencias allí citadas). La diversificación ha sido en particular una estrategia muy empleada en sistemas agrícolas, en los que tradicionalmente se ha empleado la rotación y combinación de cultivos, así como el intercalado de especies no productivas que albergan enemigos naturales de las plagas (Rao et al. 2000, Schroth et al. 2000, Gurr et al. 2004). Estrictamente en el ámbito forestal, un manual tan conocido como Dajoz (2001) ofrece como estrategias "la mezcla de clases de edad; la elección juiciosa de las especies y a mezcla de frondosas y resinosas que evite el monocultivo; el cultivo en sotobosque de plantas nectaríferas que aumenten la longevidad y la fertilidad de los parásitos entomófagos". Y, a renglón seguido, afirma que aunque tales estrategias son conocidas, en general no constituyen más que un deseo bien intencionado, y que usualmente no se aplican por exigencias socioeconómicas.

La información disponible para especies con dinámicas eruptivas similares reflejan una reducción significativa en su capacidad para formar plagas cuando el bosque se diversifica (Watt 1992, Tammaru et al. 1995, Floater y Zalucki 2000). La diversidad y estructura del bosque determina la densidad de hospedadores óptimos para la plaga, y eventualmente su capacidad de desarrollo. Si la disponibilidad de hospedadores óptimos es baja, las posibilidades de erupción se ven reducidas al depositarse mayor número de puestas en huéspedes que no permiten el desarrollo de las larvas (Tammaru et al. 1995, Floater y Zalucki 2000; ver también Masutti y Battisti 1990).

En el caso específico de la procesionaria, sabemos que mezclar los pinares con otras especies no susceptibles (frondosas, por ejemplo) reduce la tasa de ataque de las procesionarias (Jactel et al. 2011, Dulaurent et al. 2012). La mariposa puede detectar los pinos en los que ovoposita por sus emisiones de terpenos y otras sustancias, pero su capacidad para reconocer estas fuentes de emisión y, sobre todo, su capacidad para evitar interferencias de otras fuentes similares no susceptibles de ser usadas como alimento, es bastante limitada (Jactel et al. 2011). Así, manejos como los descritos suponen interferencias en la selección del lugar de ovoposición por parte de la mariposa, por lo que dificultan este proceso por el sencillo procedimiento de hacer a los individuos que componen la masa forestal menos homogéneos en su susceptibilidad a la plaga (Fig. 3). Tengamos en cuenta que la capacidad de vuelo de la mariposa de la procesionaria es usualmente pequeña (Robinet et al. 2012) y, sobre todo, que su tiempo de selección de lugar de puesta es muy limitado, ya que suele aparearse y ovopositar en la misma noche de su emergencia. Añadamos a esto que, como perteneciente a la familia Notodontidae, su capacidad auditiva es reducida en comparación con otras familias de lepidópteros nocturnos, lo que la hace muy susceptible al ataque por murciélagos mientras está en vuelo.

También sabemos que diversificar el bosque añadiendo otras especies vegetales puede ofrecer recursos alternativos (alimento y otros hospedadores) a los parasitoides de la procesionaria (Dulaurent et al. 2011b), y que algunos hábitats son inadecuados para la pupación de las larvas, lo que reduce sus poblaciones (Dulaurent et al. 2011a). Además, otros depredadores como aves pueden verse favorecidos por un adecuado mosaico de hábitats (Barbaro et al. 2008, Barbaro y Battisti 2010). Por ejemplo, abrir claros supone en principio un entorno más favorable para la procesionaria, que prefiere los bordes de rodal para desarrollarse, pero si los claros no están desnudos sino cubiertos por matorral, son entornos inadecuados para la pupación (Dulaurent et al. 2011a, Hódar et al. 2012b) y además pueden favorecer la depredación de las puestas por ortópteros efipigéridos (López-Sebastián et al. 2004, Hódar et al. 2012b). Su condición de depredadores generalistas limita su capacidad para controlar a la plaga una vez su población se ha disparado, pero pueden jugar un importante papel en el mantenimiento de la procesionaria a densidades crónicas (p. ej. Klemola et al. 2002).

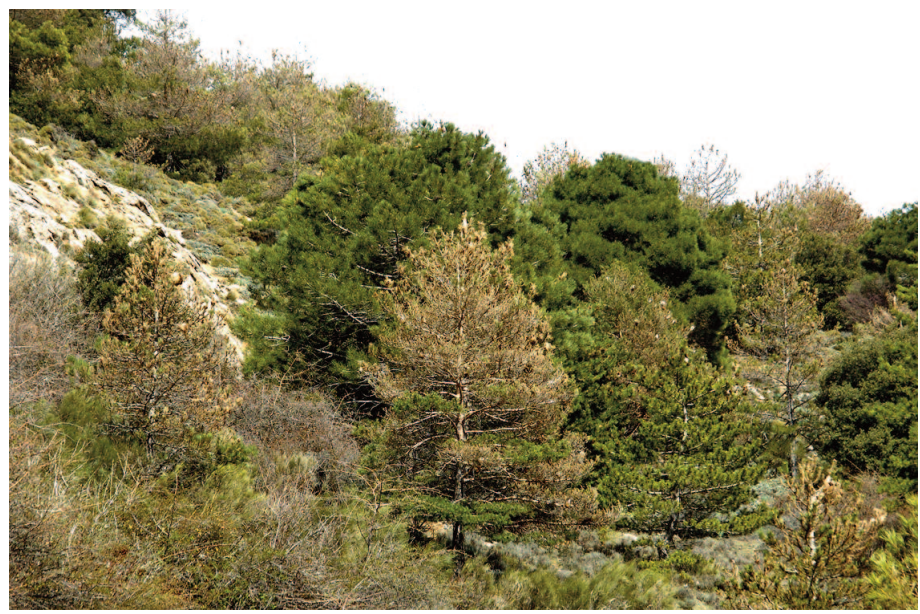

Figura 3. Incluso dentro de un mismo parche, basta con mezclar una especie de pino menos palatable (Pinus pinaster) con la susceptible (Pinus nigra) para que la defoliación del rodal disminuya. Pinar de la Cortijuela del Trevenque, Sierra Nevada, invierno de 2009.

En términos silvícolas, esto supone realizar las labores forestales necesarias para diversificar la masa, fomentando tanto la mezcla de especies arbóreas y arbustivas, como la heterogeneidad espacial de la formación forestal, sustituyendo donde fuera posible las formaciones monospecíficas por bosques mixtos de coníferas y frondosas, sin excluir el sotobosque, más heterogéneos en estructura y diversidad. En las montañas mediterráneas, los pinares cercanos a su estado natural aparecen usualmente mezclados con otras especies arbóreas (Mesón y Montoya 1993) y arbustivas, y con una baja densidad. Por lo tanto, una adecuada gestión de estas repoblaciones para hacerlas más resistentes a la procesionaria pasa por reconvertir las masas monoespecíficas y densas de las repoblaciones en bosques diversos en especies, con varios estratos de vegetación, y heterogéneos en su estructura espacial. Hoy podemos afirmar que los sistemas homogéneos son más pobres en términos de diversidad de servicios ecosistémicos y más vulnerables ante los sobresaltos ambientales. Adicionalmente, la diversidad aumenta la resiliencia del bosque, dándole la oportunidad de adaptarse a los cambios, y recuperarse tras posibles catástrofes naturales, las plagas entre ellas, a la vez que permite el uso múltiple del monte (Jones et al. 2012).

Ante estos datos, las preguntas que pueden surgir son básicamente dos: ¿Es factible? ¿Cuánto cuesta?

A la pregunta de si es factible, podríamos responder que es factible y, además, necesario. Las repoblaciones masivas con coníferas llevadas a cabo durante el siglo XX tenían mayoritariamente la intención de recuperar los ecosistemas naturales desaparecidos (Vallejo et al. 2003). Por muy diferentes razones, muchas de las labores silvícolas necesarias no se hicieron en su momento, dando lugar en muchos casos a repoblaciones densas y cerrada de pinar sin posibilidad de sucesión ni autoregeneración, susceptible a plagas y a incendios. Romper ese bloqueo de la sucesión es necesario. Esto puede conseguirse mediante actuaciones de aclareo, para permitir el establecimiento y el crecimiento de otras especies arbóreas y arbustivas bajo el dosel del pinar, algo imposible en el estado de muchas de las repoblaciones, que no obstante mantienen ese potencial (Gómez-Aparicio et al. 2009). Esta propuesta de gestión activa requiere que científicos, gestores y técnicos trabajen de forma coordinada, en el entendimiento de que sólo un trabajo en equipo puede atenuar a nivel local este "efecto colateral" provocado por los impactos globales antropogénicos.

A la pregunta de cuánto cuesta, hay que responder que probablemente haya que hacer una inversión inicial importante en la labores de manejo del hábitat, pero que se amortiza con el paso del tiempo, ya que no hay que estar continuamente interviniendo, como ocurre en la actualidad. Los aclareos y actuaciones forestales que no se llevaron a cabo hace veinte o treinta años, con árboles pe- 
queños, deben hacerse ahora en sitios con árboles más grandes y no siempre en buen estado fitosanitario, y muchas veces la madera extraída apenas permite obtener algún beneficio. Sin embargo, es una inversión inicial encaminada a que, a partir de ahí, el bosque sea más resistente y resiliente a las plagas y otros daños.

No está en nuestras manos cambiar el clima a corto plazo, es un problema global, de todo el planeta. Pero sí lo está gestionar adecuadamente la estructura de un pinar, de lo que a su vez depende la probabilidad de incidencia de plagas, haga el clima que haga. Esto nos permitiría dotarnos de ecosistemas más resilientes frente a las condiciones climáticas que nos esperan en el futuro (Sutherst et al. 2011), pero para eso tenemos que empezar a conseguirlos ya.

\section{Conclusiones}

Si queremos asegurar el futuro de nuestros bosques, no sólo tendremos que gestionarlos activamente, sino que lo tendremos que hacer bajo criterios de sostenibilidad. Además, como nuevo requerimiento, deberemos hacer una gestión que ayude o facilite la adaptación de los ecosistemas forestales a las nuevas condiciones. Por ejemplo, bajo el supuesto de una mayor frecuencia de las condiciones de sequía, un bosque menos denso (con menos pies por hectárea) puede ser más resistente, puesto que los árboles y las plantas competirían menos entre sí por un recurso como el agua. En cambio, para aumentar la resiliencia (capacidad de recuperación) de un ecosistema forestal habrá que hacer una gestión que favorezca la biodiversidad, tanto interespecífica como intraespecífica. El planteamiento de medidas de adaptación es obligado, pero sin perder de vista que la mala situación de algunos de nuestros ecosistemas se ha producido por nuestras actuaciones inadecuadas en las décadas pasadas, no por cuestiones climáticas.

El mensaje que debe calar en la sociedad es que una gestión encaminada a este fin nos dará a largo plazo bosques más capaces de enfrentarse a las incertidumbres, entre ellas climáticas, de la situación futura. Pero además, nos dará bosques cuya gestión será más barata a medio y largo plazo. Si la propia composición y estructura del bosque es capaz de atemperar la intensidad de las plagas que sufre o resulta menos susceptible a los incendios, eso que tendremos ahorrado. A corto plazo estos trabajos no son ni baratos ni fáciles, e incluso pueden ser incomprendidos por algunos sectores de la sociedad, que puede no entender que cortar árboles para hacer un aclareo es una medida drástica pero necesaria. Pero también forma parte de nuestra labor explicar a la sociedad la necesidad de estas actuaciones.

\section{Agradecimientos}

Buena parte de los resultados e ideas que expresamos en este artículo han surgido mientras trabajábamos dentro de los proyectos PROPINOL (PN22/2008) del OAPN, CONSOLIDER-MONTES (CSD2008-00040) del MICYT y GESBOME (P06-RNM-1890) de la Junta de Andalucía. Tres revisores anónimos (en especial un incisivo Revisor 2) contribuyeron a mejorar una primera versión de este manuscrito.

\section{Bibliografía}

Ayres, M. P. 1993. Global change, plant defense, and herbivory. En: Kareiva, P.M., Kingsolver, J.G., Huey, R.B. (eds.). Biotic interactions and global change, pp. 75-94. Sinauer Associates, Sunderland, Massachusets, EEUU.

Ayres, M.P., Lombardero, M.J. 2000. Assessing the consequences of global change for forest disturbance from herbivores and pathogens. Science of the Total Environment 262:263-286

Barbaro, L., Battisti, A. 2010. Birds as predators of the pine processionary moth (Lepidoptera: Notodontidae). Biological Control 56:107-114.

Barbaro, L., Couzi, L., Bretagnolle, V., Nezan, J., Vetillard, F. 2008. Multiscale habitat selection and foraging ecology of the Eurasian hoopoe (Upupa epops) in pine plantations. Biodiversity and Conservation $17: 1073-1087$
Battisti, A. 2008. Forests and climate change - lessons from insects. iForest-Biogeosciences and Forestry 1:1-5.

Battisti, A., Stastny, M., Netherer, S., Robinet, C., Schopf, A., Roques, A., Larsson, S. 2005. Expansion of geographic range in the pine processionary moth caused by increased winter temperatures. Ecological Applications 15:2084-2096.

Battisti, A., Stastny, M., Buffo, E., Larsson, S. 2006. A rapid altitudinal range expansion in the pine processionary moth produced by the 2003 climatic anomaly. Global Change Biology 12:662-671.

Berryman, A.A. 2002. Population cycles: causes and analysis. En: Berryman, A.A. (ed.) Population cycles: the case for trophic interactions, pp. 3-28 Oxford, Nueva York, EEUU.

Buffo, E., Battisti, A., Stastny, M., Larsson, S. 2007. Temperature as a predictor of survival of the pine processionary moth in the Italian Alps. Agricultural and Forest Entomology 9:65-72.

Cayuela, L., Hódar, J.A., Zamora, R. 2011. Is insecticide spraying a viable and cost-efficient management practice to control pine processionary moth in Mediterranean woodlands? Forest Ecology and Management 261:1732-1737.

Cobos-Suarez, J.M., Ruiz-Urrestarazu, M.M. 1990. Problemas fitosanitarios de la especie Pinus radiata D. Don en España, con especial referencia al País Vasco. Boletín de Sanidad Vegetal Plagas 16:37-53.

Dajoz, R. 2001. Entomología forestal. Los insectos y el bosque. MundiPrensa, Madrid.

Demolin, G. 1969a. Bioecología de la "procesionaria del pino" Thaumetopoea pityocampa Schiff. Incidencia de los factores climáticos. Boletín del Servicio de Plagas Forestales 12:9-24.

Demolin, G. 1969b. Comportement des adultes de Thaumetopoea pityocampa Schiff. Dispersion spatiale, importance écologique. Annales de Sciences Forestiéres 26:81-102.

Dulaurent, A.-M., Porté, A.J., van Halder, I., Vetillard, F., Menassieu, P., Jactel, H. 2011a. A case of habitat complementation in forest pests: Pine processionary moth pupae survive better in open areas. Forest Ecology and Management 261:1069-1076.

Dulaurent, A.-M., Rossi, J.-P., Deborde, C., Moing, A., Menassieu, P., Jactel, H. 2011b. Honeydew feeding increased the longevity of two egg parasitoids of the pine processionary moth. Journal of Applied Entomology 135:184-194.

Dulaurent, A.-M., Porté, A.J., van Halder, I., Vetillard, F., Menassieu, P., Jactel, H. 2012. Hide and seek in forests: colonization by the pine processionary moth is impeded by the presence of nonhost trees. Agricultural and Forest Entomology 14:19-27.

Floater, G.J., Zalucki, M.P. 2000. Habitat structure and egg distribution in the processionary caterpillar Ochrogaster lunifer: lessons for conservation and pest management. Journal of Applied Ecology 37:87-99.

Frisbie, R.E., Smith, J.W. Jr. 1991. Biologically intensive integrated pest management: the future. En: Menn, J.J., Steinhauer, A.L. (eds.). Progress and perspectives for the XXIst century. $170 \mathrm{pp}$. Entomological Society of America, Lanham, Maryland, EEUU.

Fuhrer, J. 2003. Agroecosystern responses to combinations of elevated $\mathrm{CO}_{2}$, ozone, and global climate change. Agriculture Ecosystems and Environment 97:1-20.

Gómez-Aparicio, L., Zavala, M.A., Bonet, F.J., Zamora, R. 2009. Are pine plantations valid tools for restoring Mediterranean forests? An assessment along abiotic and biotic gradients. Ecological Applications 19:21242141

Gurr, G.M., Scarratt, S.L., Wratten, S.D., Berndt, L., Irvin, N. 2004. Ecological engineering, habitat manipulation and pest management. En: Gurr, G.M., Wratten, S.D., Altieri, M.A. (eds.). Ecological Engineering for Pest Management, Advances in Habitat Manipulation for Arthropods, págs. 1-12. CSIRO Publishing, Australia.

Hoch, G., Toffolo, E.P., Netherer, S., Battisti, A., Schopf, A. 2009. Survival at low temperature of larvae of the pine processionary moth Thaumetopoea pityocampa from an area of range expansion. Agricultural and Forest Entomology 11:313-320.

Hódar, J.A., Zamora, R. 2004. Herbivory and climatic warming: a Mediterranean outbreaking caterpillar attacks a relict, boreal pine species. Biodiversity and Conservation 13:493-500.

Hódar, J.A., Zamora, R. 2009. Influencia de los factores abióticos y bióticos en la defoliación por la procesionaria del pino (Thaumetopoea pityocampa) en los pinares andaluces. Actas del $5^{\circ}$ Congreso Forestal Español, Ávila (España), 21-25 Septiembre 2009. SECF-Junta Castilla y León. http://www.congresoforestal.es/index.php?men=81\&par_pagina=21.

Hódar, J.A., Zamora, R., Cayuela, L. 2012a. Climate change and the incidence of a forest pest in Mediterranean ecosystems: can the North Atlantic Oscillation be used as a predictor? Climatic Change 113:699-711. 
Hódar, J.A., Torres-Muros, L., Senhadji, K., Cayuela, L., Zamora, R. 2012b. Interacción planta-herbívoro y dinámica poblacional de la procesionaria del pino en el Parque Nacional de Sierra Nevada en el marco del cambio global. En: Proyectos de investigación en Parques Nacionales: 2008-2011, págs. 387-406. Organismo Autónomo de Parques Nacionales. EGRAFSA, Madrid.

Jactel, H., Birgersson, G., Andersson, S., Schlyter, F. 2011. Non-host volatiles mediate associational resistance to the pine processionary moth. Oecologia 166:703-711.

Jactel, H., Brockerhoff, E.G. 2007. Tree diversity reduces herbivory by forest insects. Ecology Letters 10:835-848.

Jarvis, P.J. 2000. Ecological principles and environmental issues. PrenticeHall, Nueva York, EEUU.

Jones, H.P., Hole, D.G., Zavaleta, E.S. 2012. Harnessing nature to help people adapt to climate change. Nature Climate Change 2:504-509.

Keeton, W.S. 2006. Managing for late-successional/old-growth characteristics in northern hardwood-conifer forests. Forest Ecology and Management 235:129-142.

Klemola, T., Tanhuanpää, M., Korpimäki, E., Ruohomäki, K. 2002. Specialist and generalist natural enemies as an explanation for geographical gradients in population cycles of northern herbivores. Oikos 99:83-94.

Kocmankova, E., Trnka, M., Eitzinger, J., Formayer, H., Dubrovsky, M., Semerádová, D., Zalud, Z., Juroch, J., Mozny, M. 2010. Estimating the impact of climate change on the occurrence of selected pests in the Central European region. Climate Research 44:95-105.

Kogan, M. 1998. Integrated Pest Management: historical perspectives and contemporary developments. Annual Review of Entomology 43:243-270.

Kulakowski, D., Rixen, C., Bebi, P. 2006. Changes in forest structure and in the relative importance of climatic stress as a result of suppression of avalance disturbances. Forest Ecology and Management 223:66-74.

Ladanyi, M., Horvath, L. 2010. A review of the potential climate change impact on insect populations - general and agricultural aspects. Applied Ecology and Environmental Research 8:143-152.

Logan, J.A., Regniere, J., Powell, J.A. 2003. Assessing the impacts of global warming on forest pest dynamics. Frontiers in Ecology and the Environment 1:130-137.

López-Sebastián, E., García, M.D., Selfa, J. 2004. Thyreonotus corsicus corsicus y Steropleurus andalusius (Orthoptera, Tettigoniidae) como depredadores de Thaumetopoea pityocampa (Lepidoptera, Notodontidae) en el noroeste de Valencia (España). Anales de Biología 26:29-34.

Masutti, L., Battisti, A. 1990. Thaumetopoea pityocampa (Den. and Schiff.) in Italy. Bionomics and perspectives of integrated control. Journal of Applied Entomology 110:229-234.

Mesón, M., Montoya, M. 1993. Selvicultura mediterránea. Mundi-Prensa, Madrid.

Netherer, S., Schopf, A. 2008. Potential effects of climate change on insect herbivores in European forests-General aspects and the pine processionary moth as specific example. Forest Ecology and Management 259:831-838.

Newsom, L.D. 1980. The next rung up the integrated pest management ladder. Bulletin of the Entomological Society of America 26:369-374.

Petrucco-Toffolo, E., Bernardinelli, I., Stergulc, F., Battisti, A. 2006. Climate change and expansion of the Pine Processionary Moth, Thaumetopoea pityocampa, in Northern Italy. IUFRO Working Party 7.03.10
Proceedings of the Workshop 2006, Gmunden/Austria,11-14 September, 2006, pp. 331-340.

Pimentel, C., Nilsson, J.-Å. 2007. Response of Great Tits Parus major to an irruption of a Pine Processionary Moth Thaumetopoea pityocampa population with a shifted phenology. Ardea 95:191-199.

Pimentel, C., Nilsson, J.-Å. 2009. Response of passerine birds to an irruption of a pine processionary moth Thaumetopoea pityocampa population with a shifted phenology. Ardeola 56:189-203.

Pimentel, D., Perkins, J.H. (eds). 1980. Pest control: cultural and environmental aspects. AAAS Selected Symposium, Westview Press. Boulder, Colorado, EEUU. 243 pp.

Rao, M.R., Singh, M.P., Day, R. 2000. Insect pest problems in tropical agroforestry systems: contributory factors and strategies for management. Agroforestry Systems 50: 243-277.

Robinet, C. 2006. Mathematical modelling of invasion processes in ecology: the pine processionary moth as a case study. PhD thesis, EHESS, Paris. France.

Robinet, C., Baier, P., Pennerstorfer, J., Schopf, A., Roques, A. 2007. Modelling the effects of climate change on the potential feeding activity of Thaumetopoea pityocampa (Den and Schiff.) (Lep., Notodontidae) in France. Global Ecology and Biogeography 16:460-471.

Robinet, C., Imbert, C.-E., Rousselet, J., Sauvard, D., Garcia, J., Goussard, F., Roques, A. 2012. Human-mediated long-distance jumps of the pine processionary moth in Europe. Biological Invasions 14: 1557-1569.

Roques, L., Soubeyrand, S., Rousselet, J. 2011. A statistical-reaction-diffusion approach for analyzing expansion processes. Journal of Theoretical Biology 274:43-51.

Schowalter, T.D. 1986. Ecological strategies of forest insects: the need for a community-level approach to reforestation. New Forests 1:57-66.

Schroth, G., Krauss, U., Gasparotto, L., Aguilar, J., Duarte, A., Vohland, K. 2000. Pests and diseases in agroforestry systems of the humid tropics. Agroforestry Systems 50:199-241.

Sutherst, R.W., Constable, F., Finlay, K.J., Harrington, R., Luck, J., Zalucki, M.P. 2011. Adapting to crop pest and pathogen risks under a changing climate. WIREs-Climate Change 2:220-237.

Tammaru, T. Kaitaniemi, P., Ruohomäki, K. 1995. Oviposition choices of Epirrita autumnata (Lepidoptera: Geometridae) in relation to its eruptive population dynamics. Oikos 74:296-304.

Vallejo, R., Cortina, J., Vilagrosa, A., Seva, J.P., Alloza, J.A. 2003. Problemas y perspectivas de la utilización de leñosas autóctonas en la restauración forestal. En: Rey-Benayas, J.M., Espigares. T., Nicolau, J.M (eds.). Restauración de ecosistemas mediterráneos, pp. 11-42. UAH. Álcalá de Henares. España.

Vega, J.M., Moneo, I., Ortiz, J.C., Palla, P.S., Sanchís, M.E., Vega, J., Gonzalez-Muñoz, M., Battisti, A., Roques, A. 2011. Prevalence of cutaneous reactions to the pine processionary moth (Thaumetopoea pityocampa) in an adult population. Contact Dermatitis 64:220-2288.

Watt, A. D. 1992. Insect pest population dynamics: effects of tree species diversity. En: Cannell, M.G.R., Malcolm, D.C., Robertson, P.A. (eds.). The ecology of mixed-species stands of trees, pp. 267-275. Blackwell, Oxford, Reino Unido.

Williams, D.W., Liebhold, A.M. 1995. Herbivorous insects and global change: Potential changes in the spatial distribution of forest defoliator outbreaks. Journal of Biogeography 22:665-671. 